\title{
Computation of Inertial Forces and Torques Associated with
}

\section{Flapping Wings}

\author{
Michael W. Oppenheimer* \\ Shorya Awtar ${ }^{\dagger}$ \\ David O. Sigthorsson $\ddagger$ \\ Isaac E. Weintraub $\S$ \\ David B. Doman ฯ
}

\begin{abstract}
Typically, inertial loads associated with flapping wing micro air vehicles are ignored for analysis and control law development purposes. The goal of this work is to compute the inertial loads associated with flapping a wing and compare those loads to aerodynamic loads. It is assumed that the wing is a rigid flat plate so that there is no in-plane, out-of-plane, or torsional bending of the wing. The analysis begins by computing the acceleration of the wing center-of-gravity over a complete wingbeat cycle. Newton's second law is then used to compute the inertial forces and eventually, the inertial moments. Simulation results are provided which compare the magnitude of the inertial loads to the aerodynamic loads for a wing of varying geometry.
\end{abstract}

*Senior Electronics Engineer, Control Sciences Branch, 2210 Eighth Street, Bldg. 146, Rm. 305, Air Force Research Laboratory, WPAFB, OH 45433-7531 Email: Michael.Oppenheimer@wpafb.af.mil, Ph. (937) 2558490, Fax (937) 656-4000, Associate Fellow, AIAA

$\dagger$ Assistant Professor Mechanical Engineering, 2268 George G. Brown Laboratory, 2350 Hayward St., Ann Arbor, MI 48109-2125

${ }_{\ddagger}^{\ddagger}$ Principal Engineer, General Dynamics Information Technology, 2210 Eighth Street, Bldg. 146, Rm. 304A, WPAFB, OH 45433-7531 Email: David.Sigthorsson@gdit.com, Ph. (937) 255-9707, Fax (937) 6564000, Member, AIAA

§Principal Engineer, General Dynamics Information Technology, 2210 Eighth Street, Bldg. 146, Rm. 305, WPAFB, OH 45433-7531 Email: Isaac.Weintraub@gdit.com, Ph. (937) 255-3110, Fax (937) 656-4000, Member, AIAA

`Principal Aerospace Engineer, Control Sciences Branch, 2210 Eighth Street, Bldg. 146, Rm. 305, Air Force Research Laboratory, WPAFB, OH 45433-7531 Email david.doman@wpafb.af.mil, Ph. (937) 255-8451, Fax (937) 656-4000, Fellow AIAA 


\section{Introduction}

Flapping wing micro air vehicles (MAVs) have received a great deal of interest from the research community due to their potential to achieve insect-like maneuverability. ${ }^{1-7}$ The ability to mimic the flight behavior of insects could enable such a vehicle to perform missions which larger, fixed wing vehicles are unable to perform, such as intelligence, surveillance, and reconnaissance in urban environments and indoor locations. These potential capabilities have prompted continued research into flapping wing flight dynamics, control law development, ${ }^{8,9}$ and fabrication..$^{3,4}$

The aerodynamics of flapping wing flight are complex. ${ }^{5,10-18}$ Wing-wing interactions, wake capture, leading-edge vortices, wing rotation, and acceleration are examples of some of the complex phenomena that occur. This work in focused on calculating the inertial forces associated with the flapping wings. Typically, the inertial forces are ignored and only the aerodynamic forces due to wing flapping are considered. This work aims to determine the magnitude of the wing inertial forces and compare the inertial to aerodynamic forces and moments. It is assumed that the wing is a rigid flat plate. Therefore, there is no in-plane, out-of-plane, or torsional bending of the wing. Additionally, the wing flip dynamics are ignored in this analysis and it is assumed that the wing instantaneously flips at the end of each stroke and the flip motion does not contribute any force or moment.

The vehicle selected for this study, shown in Figure 1, is similar to the Harvard RoboFly. ${ }^{3}$ The wings are assumed to be triangular in shape. During the simulation runs, the wing geometry is altered by varying the chord, however, the wings always retain the triangular shape.

This paper is organized as follows: Section II discusses notation that is used throughout the manuscript. In particular, the coordinate frames used to compute the forces and moments are provided. In Section III, the acceleration of the centers-of-gravity of the wings are computed. Section IV contains the computation of the inertial forces and moments. Section V contains simulation results, which compare the inertial and aerodynamic forces and moments, and conclusions are provided in Section VI.

\section{Notation}

In this section, some basic notation that is used throughout this work is introduced. First, the wingbeat forcing function is described. Following this, coordinate frames and rotation matrices are described.

Let the wingbeat forcing function, which describes the motion of the wing, be

$$
\phi(t)=\cos (\omega t)
$$




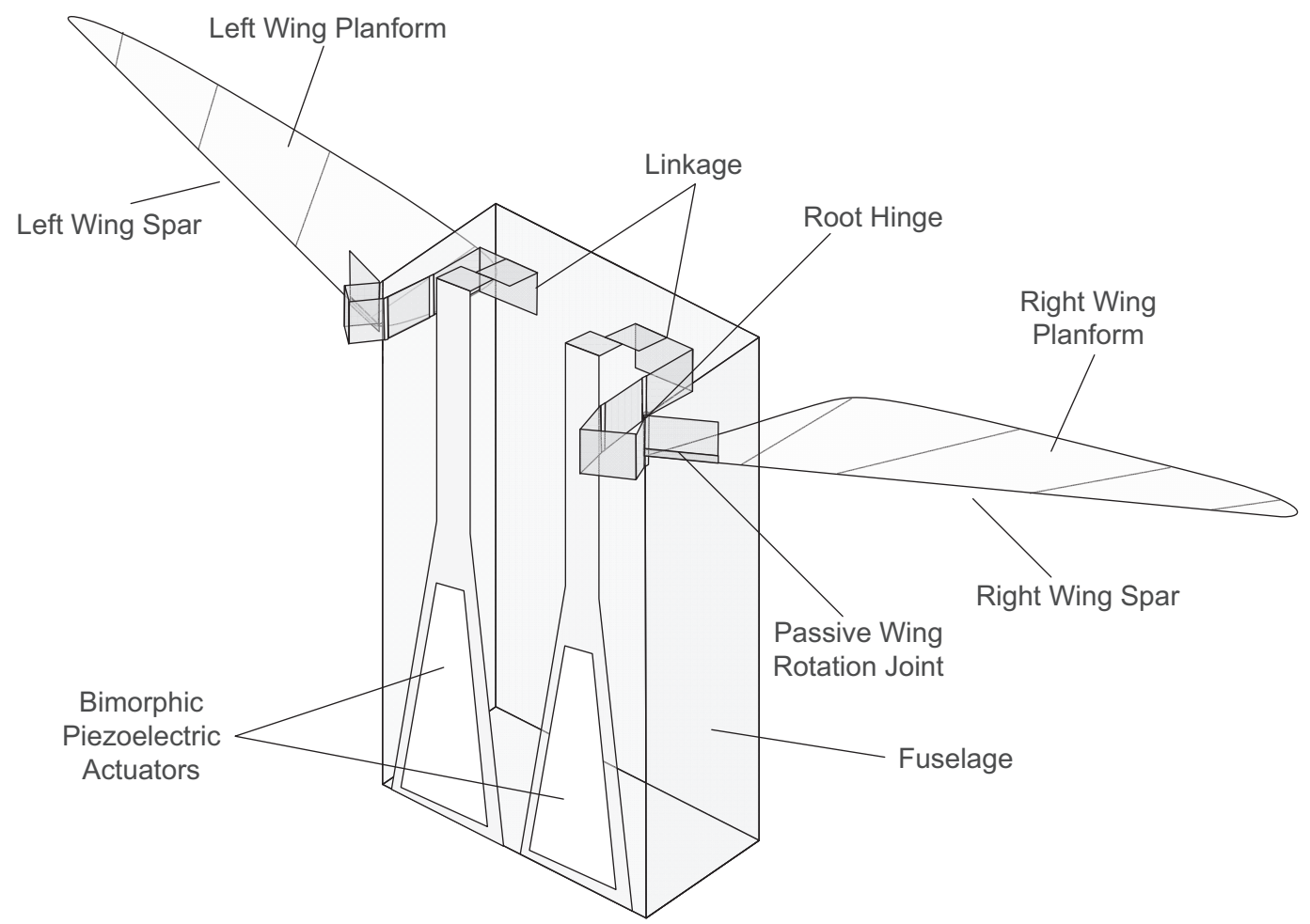

Figure 1: General assembly of a minimally actuated flapping wing micro air vehicle.

Figure 2 shows one cycle of the wingbeat forcing function, time derivative of $\phi(t)$, and the second time derivative of $\phi(t)$, along with the portions that correspond to the upstroke and downstroke. A complete cycle of wingbeat is defined as an upstroke followed by a downstroke. In order to simplify notation, let

$$
k=\left\{\begin{array}{cc}
1 & \text { Downstroke } \\
-1 & \text { Upstroke }
\end{array}\right.
$$

There are nine coordinate frames for this vehicle, as shown in Figure 3. The body frame is centered at the center-of-gravity of the entire vehicle (excluding the wings), with the body $x$ axis pointed up, the body $y$ axis pointed out the right wing, and the body $z$ axis pointed out the front of the vehicle. The body axes are denoted with the subscript $B$. Define the right and left wing root frames. These two frames are always aligned with the body frame and are located at the right and left wing attachment, or wing root, points. The subscripts $R W R$ and $L W R$ denote the right and left wing root frames. Additionally, define right and left wing spar frames (denoted by the subscripts 'RWS' and 'LWS'). The spar frames are centered at the wing root and are thus coincident with the wing root frames. The $y$ axis of the right wing spar frame points along the right wing spar, while the $y$ axis of the left 


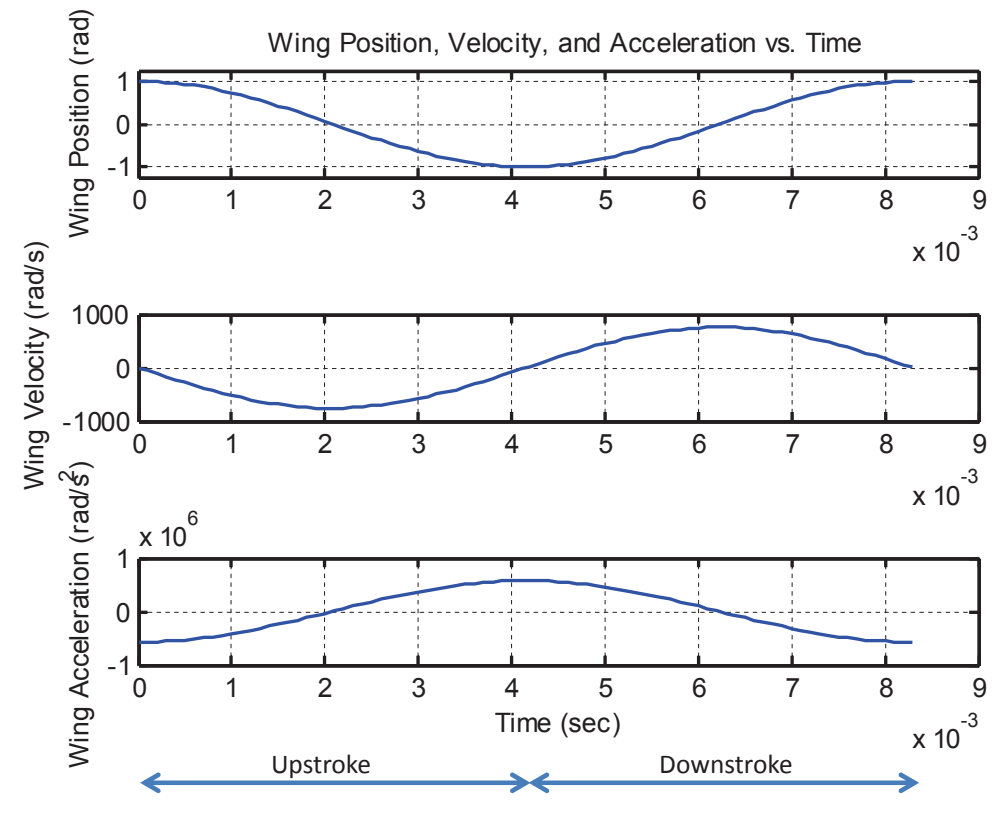

Figure 2: $\phi(t), \dot{\phi}(t)$, and $\ddot{\phi}(t)$.

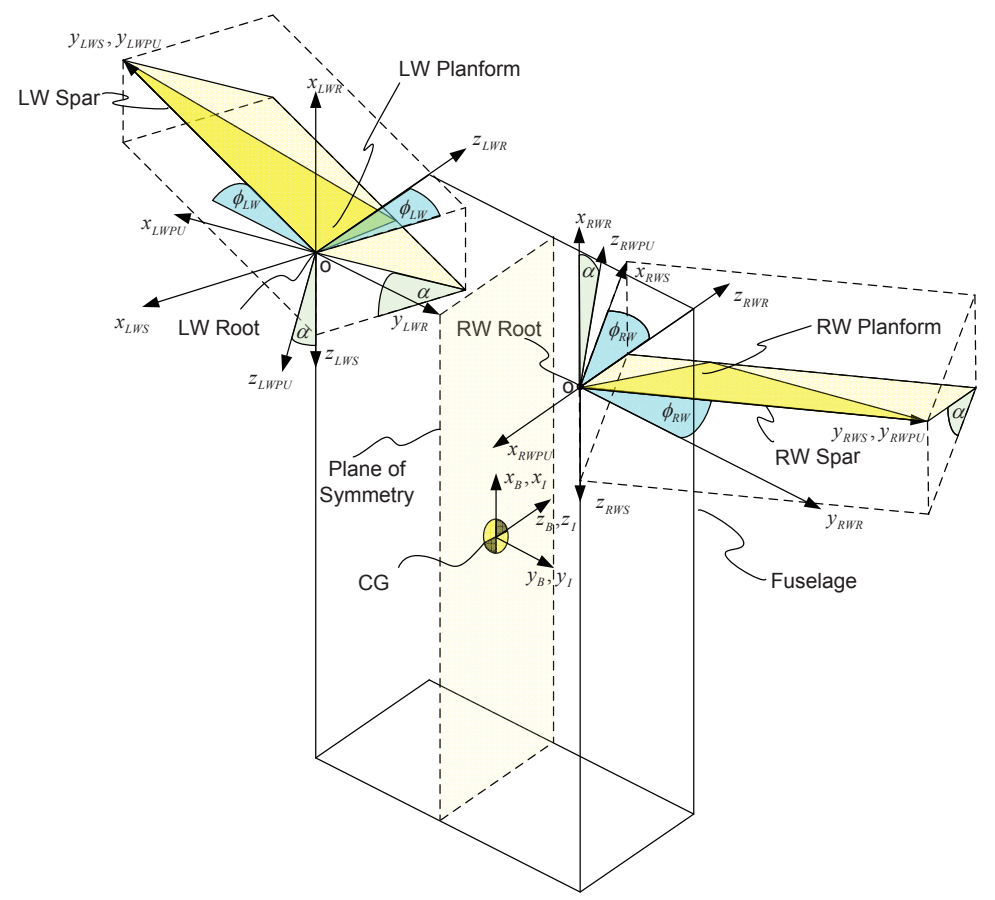

Figure 3: Coordinate frames.

wing spar frame points along the left wing spar and originates at the left wing root. The $z$ axis of both the left and right spar frames points in the $-z_{B}$ direction. The spar frame 
rotates with the wing and the angle between the $y_{R W R}$ axis and the $y_{R W S}$ axis is $\phi_{R W}(t)$. The same exists for the left wing. The last set of coordinate frames are the wing planform frames. There are wing planforms frames for the left and right wings and the upstroke and downstroke (4 wing planform frames total). The right wing planform up ( $R W P U)$ frame has it $y$ axis pointing from the wing root along the wing, and, for zero wing angle of attack, the $x$ axis points back $\left(-z_{B}\right.$ direction) and the $z$ axis points up ( $x_{B}$ direction). When the wing angle of attack is nonzero, a rotation about $y_{R W P U}$ occurs so that the wing is contained in the $x_{R W P U}, y_{R W P U}$ frame. The right wing planform downstroke $(R W P D)$ is similar to its upstroke counterpart, except the $x_{R W P D}$ axis points in the $z_{B}$ direction for zero wing angle of attack and the $z_{R W P D}$ points in the $-x_{B}$ direction. Similar definitions exist for the left wing. The relationships between the spar and wing planform frames are described in Figure 4, where $\alpha$ is the wing angle-of-attack.

The transformations from the wing planform frames to the spar frames are

$$
\mathbf{R}_{R W P 2 R W S}=\left(\begin{array}{ccc}
k \cos \alpha & 0 & \sin \alpha \\
0 & 1 & 0 \\
-\sin \alpha & 0 & k \cos \alpha
\end{array}\right)
$$

and

$$
\mathbf{R}_{L W P 2 L W S}=\left(\begin{array}{ccc}
-k \cos \alpha & 0 & \sin \alpha \\
0 & 1 & 0 \\
-\sin \alpha & 0 & -k \cos \alpha
\end{array}\right)
$$

where the subscripts $R W P 2 R W S$ and $L W P 2 L W S$ define right wing planform to right spar and left wing planform to left spar. The transformations from the spar frames to the wing root frames are

$$
\mathbf{R}_{R W S 2 R W R}=\left(\begin{array}{ccc}
0 & 0 & -1 \\
-\sin \phi_{R W} & \cos \phi_{R W} & 0 \\
\cos \phi_{R W} & \sin \phi_{R W} & 0
\end{array}\right)
$$

and

$$
\mathbf{R}_{L W S 2 L W R}=\left(\begin{array}{ccc}
0 & 0 & -1 \\
-\sin \phi_{L W} & -\cos \phi_{L W} & 0 \\
-\cos \phi_{L W} & \sin \phi_{L W} & 0
\end{array}\right)
$$

Since the right and left wing root frames are always aligned with the body frame,

$$
\mathbf{R}_{R W R 2 B}=\mathbf{R}_{L W R 2 B}=\mathbf{I}
$$



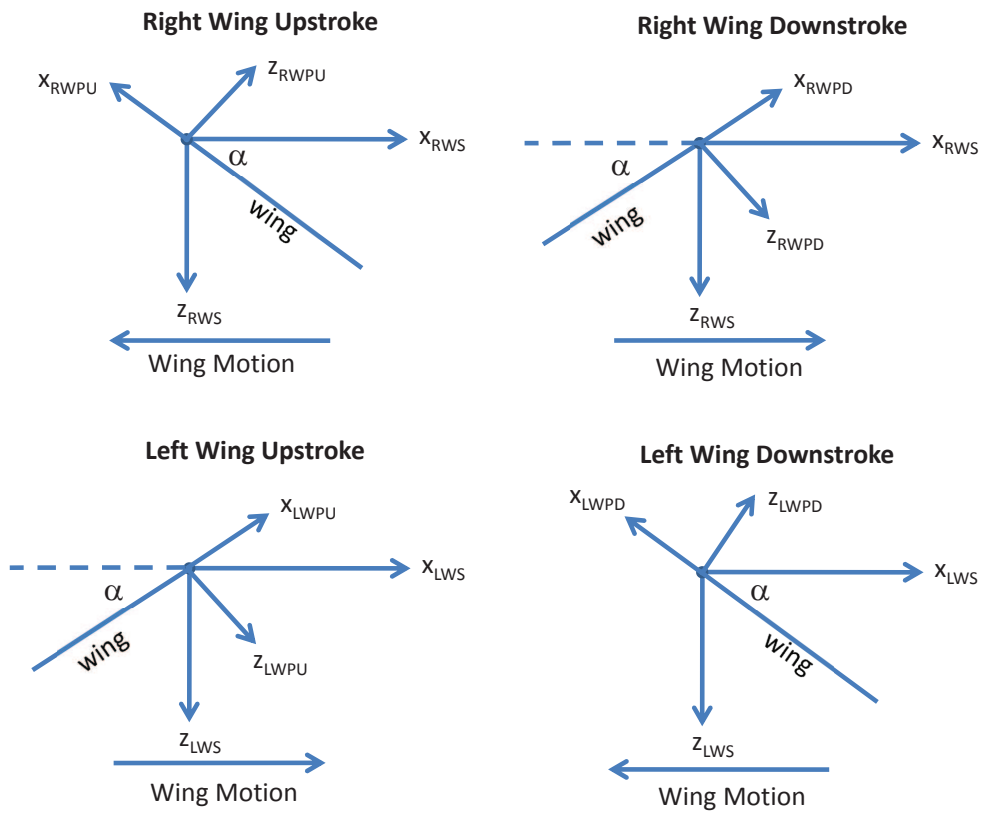

Figure 4: Wing planform and wing spar coordinate frames.

\section{Acceleration of Wing Center-of-Gravity}

In this section, the acceleration of each wing center-of-gravity is computed. Once the acceleration is obtained, Newton's second law is used to compute the inertial forces.

To begin, the position vectors from the origin of the wing planform frames to the centersof-gravity, of each wing, are computed. The geometry is shown in Figure 5. From this figure, it can be seen that

$$
\mathbf{r}_{R W c g / R W P U}^{R W P U}=\mathbf{r}_{R W c g / R W P D}^{R W P D}=\mathbf{r}_{L W c g / L W P U}^{L W P U}=\mathbf{r}_{L W c g / L W P D}^{L W P D}=\left[\begin{array}{c}
-x \\
y \\
0
\end{array}\right]
$$

where the superscripts define the coordinate frame in which the vector is written and the subscript defines the position vector starting point (origin of $R W P U, R W P D, L W P U$, or $L W P D$ frames) and the final point of the position vector ( $R W c g$ or $L W c g$ ). Therefore, the subscript notation $R W c g / R W P U$ means the right wing center-of-gravity with respect to the origin of the $R W P U$ frame. Using the transformations in Equations 3 and 4, the position 
vectors in Equation 8 can be expressed in the spar frames as

$$
\begin{aligned}
& \mathbf{r}_{R W c g / R W P U}^{R W S}=\mathbf{R}_{R W P 2 R W S} \mathbf{r}_{R W c g / R W P U}^{R W P U}=\left[\begin{array}{c}
\cos (\alpha) x \\
y \\
\sin \alpha
\end{array}\right] \\
& \mathbf{r}_{R W c g / R W P D}^{R W S}=\mathbf{R}_{R W P 2 R W S} \mathbf{r}_{R W c g / R W P D}^{R W P D}=\left[\begin{array}{c}
-\cos (\alpha) x \\
y \\
\sin \alpha
\end{array}\right] \\
& \begin{array}{l}
\mathbf{r}_{L W c g / L W P U}^{L W S}=\mathbf{R}_{L W P 2 L W S} \mathbf{r}_{L W c g / L W P U}^{L W P U}=\left[\begin{array}{c}
-\cos (\alpha) x \\
y \\
\sin \alpha
\end{array}\right] \\
\mathbf{r}_{L W c g / L W P D}^{L W S}=\mathbf{R}_{L W P 2 L W S} \mathbf{r}_{L W c g / L W P D}^{L W P D}=\left[\begin{array}{c}
\cos (\alpha) x \\
y \\
\sin \alpha
\end{array}\right]
\end{array}
\end{aligned}
$$

\section{Right Wing Downstroke $\quad$ Right Wing Upstroke}

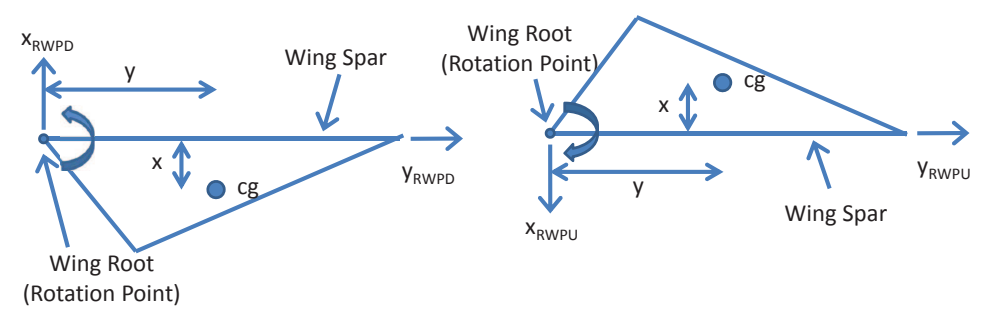

Left Wing Downstroke
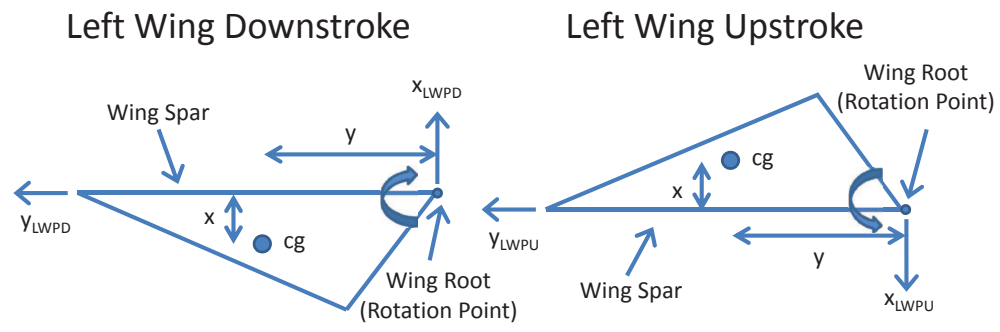

Figure 5: Wing center-of-gravity definitions.

The wing has a passive rotation joint that fixes the wing angle of attack during each stroke. It is assumed that the wing instantaneously flips at the end of each stroke. Therefore, wing flip dynamics are ignored. Additionally, it is assumed that the instantaneous wing flip does not contribute to the generation of aerodynamic or inertial forces and moments.

The angular velocity of the coordinate frames is now computed. Since the wing flip dynamics are ignored, there is no acceleration between the wing planform and spar frames. 
Hence,

$$
\boldsymbol{\omega}_{R W P U / R W S}=\boldsymbol{\omega}_{R W P D / R W S}=\boldsymbol{\omega}_{L W P U / L W S}=\boldsymbol{\omega}_{L W P D / L W S}=\left[\begin{array}{l}
0 \\
0 \\
0
\end{array}\right]
$$

where the subscript 'RWPU/RWS' describes the angular velocity of the right wing planform upstroke frame with respect to the right wing spar frame. The angular velocity of the spar frames with respect to the root and body frames are

$$
\begin{aligned}
& \boldsymbol{\omega}_{R W S / R W R}^{R W S}=\left[\begin{array}{c}
0 \\
0 \\
-\dot{\phi}_{R W}
\end{array}\right] \\
& \boldsymbol{\omega}_{R W S / B}^{B}=\left[\begin{array}{c}
\dot{\phi}_{R W} \\
0 \\
0
\end{array}\right] \\
& \boldsymbol{\omega}_{L W S / L W R}^{L W S}=\left[\begin{array}{c}
0 \\
0 \\
\dot{\phi}_{L W}
\end{array}\right] \\
& \boldsymbol{\omega}_{L W S / B}^{B}=\left[\begin{array}{c}
-\dot{\phi}_{L W} \\
0 \\
0
\end{array}\right]
\end{aligned}
$$

where, as before, the superscript denotes the frame in which the vector is written. Consider the first entry in Equation 11. It appears that the angular velocity of the right wing spar with respect to the right wing root, written in the right wing spar frame, is negative about the $z$ axis of the right wing spar frame. However, from Figure 2, it can be seen that $\dot{\phi}$ is negative on the upstroke. Hence, the right wing angular velocity is actually positive during the upstroke and negative during the downstroke, as expected.

At this point, the velocity and acceleration of the wing center-of-gravity can be computed. The velocity is computed using the equation of Coriolis ${ }^{19}$ such that

$$
\begin{gathered}
\mathbf{v}_{R W c g_{\text {up }}}^{R W S}=\mathbf{v}_{R W R}+\boldsymbol{\omega}_{R W S / R W R}^{R W S} \times \mathbf{r}_{R W c g / R W P U}^{R W S} \\
\mathbf{v}_{R W c g_{\text {down }}^{R W}}^{R W S}=\mathbf{v}_{R W R}+\boldsymbol{\omega}_{R W S / R W R}^{R W S} \times \mathbf{r}_{R W c g / R W P D}^{R W S} \\
\mathbf{v}_{L W c g_{\text {up }}}^{L W W}=\mathbf{v}_{L W R}+\boldsymbol{\omega}_{L W S / L W R}^{L W S} \times \mathbf{r}_{L W c g / L W P U}^{L W S} \\
\mathbf{v}_{L W c g_{\text {down }} W W}^{L W S}=\mathbf{v}_{L W R}+\boldsymbol{\omega}_{L W S / L W R}^{L W S} \times \mathbf{r}_{L W c g / L W P D}^{L W S}
\end{gathered}
$$

Assuming the vehicle to be in pure hover, the velocity of the right and left wing roots are 
zero. Therefore, $\mathbf{v}_{R W R}=\mathbf{v}_{L W R}=\left[\begin{array}{lll}0 & 0 & 0\end{array}\right]$. Using Equations 11 and 9 in Equation 12 yields

$$
\mathbf{v}_{R W c g}^{R W S}=\left[\begin{array}{c}
y \dot{\phi}_{R W} \\
k \cos (\alpha) x \dot{\phi}_{R W} \\
0
\end{array}\right] \mathbf{v}_{L W c g}^{L W S}=\left[\begin{array}{c}
-y \dot{\phi}_{L W} \\
k \cos (\alpha) x \dot{\phi}_{L W} \\
0
\end{array}\right]
$$

Equation 13 holds for both the upstroke $(k=-1)$ and the downstroke $(k=1)$ and describes the linear velocity of the right (left) wing center-of-gravity written in the right (left) spar frame.

In a similar fashion, the acceleration of the wing center-of-gravity is

$$
\begin{aligned}
& \mathbf{a}_{R W c g_{u p}}^{R W S}=\mathbf{a}_{R W R}+\boldsymbol{\omega}_{R W S / R W R}^{R W S} \times\left(\boldsymbol{\omega}_{R W S / R W R}^{R W S} \mathbf{r}_{R W c g / R W P U}^{R W S}\right)+\boldsymbol{\alpha}_{R W S / R W R}^{R W S} \times \mathbf{r}_{R W c g / R W P U}^{R W S} \\
& \mathbf{a}_{R W c g_{\text {down }}}^{R W S}=\mathbf{a}_{R W R}+\boldsymbol{\omega}_{R W S / R W R}^{R W S} \times\left(\boldsymbol{\omega}_{R W S / R W R}^{R W S} \mathbf{r}_{R W c g / R W P D}^{R W S}\right)+\boldsymbol{\alpha}_{R W S / R W R}^{R W S} \times \mathbf{r}_{R W c g / R W P D}^{R W S} \\
& \mathbf{a}_{L W c g_{u p}}^{L W S}=\mathbf{a}_{L W R}+\boldsymbol{\omega}_{L W S / L W R}^{L W S} \times\left(\boldsymbol{\omega}_{L W S / L W R}^{L W S} \mathbf{r}_{L W c g / L W P U}^{L W S}\right)+\boldsymbol{\alpha}_{L W S / L W R}^{L W S} \times \mathbf{r}_{L W c g / L W P U}^{L W S} \\
& \mathbf{a}_{L W c g_{\text {down }}}^{L W S}=\mathbf{a}_{L W R}+\boldsymbol{\omega}_{L W S / L W R}^{L W S} \times\left(\boldsymbol{\omega}_{L W S / L W R}^{L W S} \mathbf{r}_{L W c g / L W P D}^{L W S}\right)+\boldsymbol{\alpha}_{L W S / L W R}^{L W S} \times \mathbf{r}_{L W c g / L W P D}^{L W S}
\end{aligned}
$$

As with the velocity, the acceleration of the right and left wing roots is zero when the vehicle is hovering, hence, $\mathbf{a}_{R W R}=\mathbf{a}_{L W R}=\left[\begin{array}{lll}0 & 0 & 0\end{array}\right]$. Performing the operations in Equation 14 produces

$$
\begin{gathered}
\mathbf{a}_{R W c g}^{R W S}=\left[\begin{array}{c}
k \cos (\alpha) x \dot{\phi}^{2}+y \ddot{\phi} \\
k \cos (\alpha) x \ddot{\phi}-y \dot{\phi}^{2} \\
0
\end{array}\right] \\
\mathbf{a}_{L W c g}^{L W S}=\left[\begin{array}{c}
-k \cos (\alpha) x \dot{\phi}^{2}-y \ddot{\phi} \\
k \cos (\alpha) x \ddot{\phi}-y \dot{\phi}^{2} \\
0
\end{array}\right]
\end{gathered}
$$

Equation 15 holds for both the upstroke $(k=-1)$ and the downstroke $(k=1)$. From Equation 15, it can be seen that the acceleration of the wing center-of-gravity is a function of both the wing velocity and the wing acceleration.

\section{Wing Inertial Forces and Moments}

In the previous section, the acceleration of the center-of-gravity of each wing was computed. These accelerations will now be used to compute the inertial loads. The inertial loads are simply the negative of Newton's second law. Therefore, the inertial forces are

$$
\mathbf{F}_{I_{R W}}^{R W S}=-m_{R W} a_{R W c g}^{R W S} \quad \mathbf{F}_{I_{L W}}^{L W S}=-m_{L W} a_{L W c g}^{L W S}
$$


where $m_{R W}, m_{L W}$ are the masses of the right and left wings, respectively. Using the accelerations in Equation 15, the inertial forces become

$$
\begin{aligned}
\mathbf{F}_{I_{R W}}^{R W S} & =\left[\begin{array}{c}
-m_{R W}\left(k \cos (\alpha) x \dot{\phi}_{R W}^{2}+y \ddot{\phi}_{R W}\right) \\
-m_{R W}\left(k \cos (\alpha) x \ddot{\phi}_{R W}-y \dot{\phi}_{R W}^{2}\right) \\
0
\end{array}\right] \\
\mathbf{F}_{I_{L W}}^{L W S} & =\left[\begin{array}{c}
-m_{L W}\left(-k \cos (\alpha) x \dot{\phi}_{L W}^{2}-y \ddot{\phi}_{L W}\right) \\
-m_{L W}\left(k \cos (\alpha) x \ddot{\phi}_{L W}-y \dot{\phi}_{L W}^{2}\right) \\
0
\end{array}\right]
\end{aligned}
$$

One of the objectives of this work is to compare the inertial loads with the aerodynamic loads. The aerodynamics forces are written in the body frame. Therefore, the inertial forces in Equation 17 need to be transformed to the body frame. Using Equations 5, 6, and 7, the inertial forces, written in the body coordinate frame, are

$$
\begin{aligned}
\mathbf{F}_{I_{R W}}^{B}= & {\left[\begin{array}{c}
0 \\
m_{R W}\left\{\left(k \cos (\alpha) x \dot{\phi}_{R W}^{2}+y \ddot{\phi}_{R W}\right) \sin \phi_{R W}-\left(k \cos (\alpha) x \ddot{\phi}_{R W}-y \dot{\phi}_{R W}^{2}\right) \cos \phi_{R W}\right\} \\
-m_{R W}\left\{\left(k \cos (\alpha) x \dot{\phi}_{R W}^{2}+y \ddot{\phi}_{R W}\right) \cos \phi_{R W}+\left(k \cos (\alpha) x \ddot{\phi}_{R W}-y \dot{\phi}_{R W}^{2}\right) \sin \phi_{R W}\right\}
\end{array}\right] } \\
\mathbf{F}_{I_{L W}}^{B}= & {\left[\begin{array}{c}
0 \\
-m_{L W}\left\{\left(k \cos (\alpha) x \dot{\phi}_{L W}^{2}+y \ddot{\phi}_{L W}\right) \sin \phi_{L W}-\left(k \cos (\alpha) x \ddot{\phi}_{L W}-y \dot{\phi}_{L W}^{2}\right) \cos \phi_{L W}\right\} \\
-m_{L W}\left\{\left(k \cos (\alpha) x \dot{\phi}_{L W}^{2}+y \ddot{\phi}_{L W}\right) \cos \phi_{L W}+\left(k \cos (\alpha) x \ddot{\phi}_{L W}-y \dot{\phi}_{L W}^{2}\right) \sin \phi_{L W}\right\}
\end{array}\right] }
\end{aligned}
$$

Equation 18 provides one of the desired results, the inertial forces due to wing flapping written in the body coordinate system.

In order to compute the moments about the body frame axes, the position vector from the center-of-gravity of the vehicle (origin of the body coordinate frame) to the center-of-gravity of each wing is needed. Equation 8 provides the position vectors from the center-of-gravity of each wing to the right and left wing planform frame origins. Transforming these position 
vectors into the body frame, using the rotation matrices in Equations 3, 4, 5, and 6, yields

$$
\begin{aligned}
& \mathbf{r}_{R W c g / R W R}^{B}=\mathbf{R}_{R W S 2 B} \mathbf{R}_{R W P 2 R W S}\left[\begin{array}{c}
-x \\
y \\
0
\end{array}\right]=\left[\begin{array}{c}
-x \sin (\alpha) \\
k \cos (\alpha) \sin \left(\phi_{R W}\right) x+\cos \left(\phi_{R W}\right) y \\
-k \cos (\alpha) \cos \left(\phi_{R W}\right) x+\sin \left(\phi_{R W}\right) y
\end{array}\right] \\
& -x \sin (\alpha) \\
& \mathbf{r}_{L W c g / L W R}^{B}=\mathbf{R}_{L W S 2 B} \mathbf{R}_{L W P 2 L W S}\left[\begin{array}{c}
-x \\
y \\
0
\end{array}\right]=\left[\begin{array}{c}
-k \cos (\alpha) \sin \left(\phi_{L W}\right) x-\cos \left(\phi_{L W}\right) y \\
-k \cos (\alpha) \cos \left(\phi_{L W}\right) x+\sin \left(\phi_{L W}\right) y
\end{array}\right]
\end{aligned}
$$

The position vectors from the origin of the body axes to the origins of the right and left wing root frames, written in body axes, are

$$
\mathbf{r}_{R W R / B}^{B}=\left[\begin{array}{c}
x_{R W R} \\
y_{R W R} \\
z_{R W R}
\end{array}\right] \mathbf{r}_{L W R / B}^{B}=\left[\begin{array}{c}
x_{L W R} \\
y_{L W R} \\
z_{L W R}
\end{array}\right]
$$

Note that for wing roots that are above the vehicle center-of-gravity and attached at the same $x$ body axis location, $x_{R W R}=x_{L W R}>0$. Also, for a vehicle which has a plane of symmetry in the $x_{B}, z_{B}$ plane, $y_{R W R}=-y_{L W R}$ with $y_{R W R}>0$. Typically, the wings are attached at the same $z_{B}$ location so that $z_{R W R}=z_{L W R}$. Now, the position vectors from the vehicle center-of-gravity to each wing's center-of-gravity become

$$
\begin{aligned}
& \mathbf{r}_{R W c g / B}^{B}=\mathbf{r}_{R W R / B}^{B}+\mathbf{r}_{R W c g / R W R}^{B}= {\left[\begin{array}{c}
x_{R W R}-x \sin (\alpha) \\
y_{R W R}+k \cos (\alpha) \sin \left(\phi_{R W}\right) x+\cos \left(\phi_{R W}\right) y \\
z_{R W R}-k \cos (\alpha) \cos \left(\phi_{R W}\right) x+\sin \left(\phi_{R W}\right) y
\end{array}\right] } \\
& \mathbf{r}_{L W c g / B}^{B}=\mathbf{r}_{L W R / B}^{B}+\mathbf{r}_{L W c g / L W R}^{B}=\left[\begin{array}{c}
x_{L W R}-x \sin (\alpha) \\
y_{L W R}-k \cos (\alpha) \sin \left(\phi_{L W}\right) x-\cos \left(\phi_{L W}\right) y \\
z_{L W R}-k \cos (\alpha) \cos \left(\phi_{L W}\right) x+\sin \left(\phi_{L W}\right) y
\end{array}\right]
\end{aligned}
$$

Then, the inertial moments can be calculated with a cross-product operation

$$
\begin{aligned}
& \mathbf{M}_{I_{R W}}=\mathbf{r}_{R W c g / B}^{B} \times \mathbf{F}_{I_{R W}}^{B} \\
& \mathbf{M}_{I_{L W}}=\mathbf{r}_{L W c g / B}^{B} \times \mathbf{F}_{I_{L W}}^{B}
\end{aligned}
$$




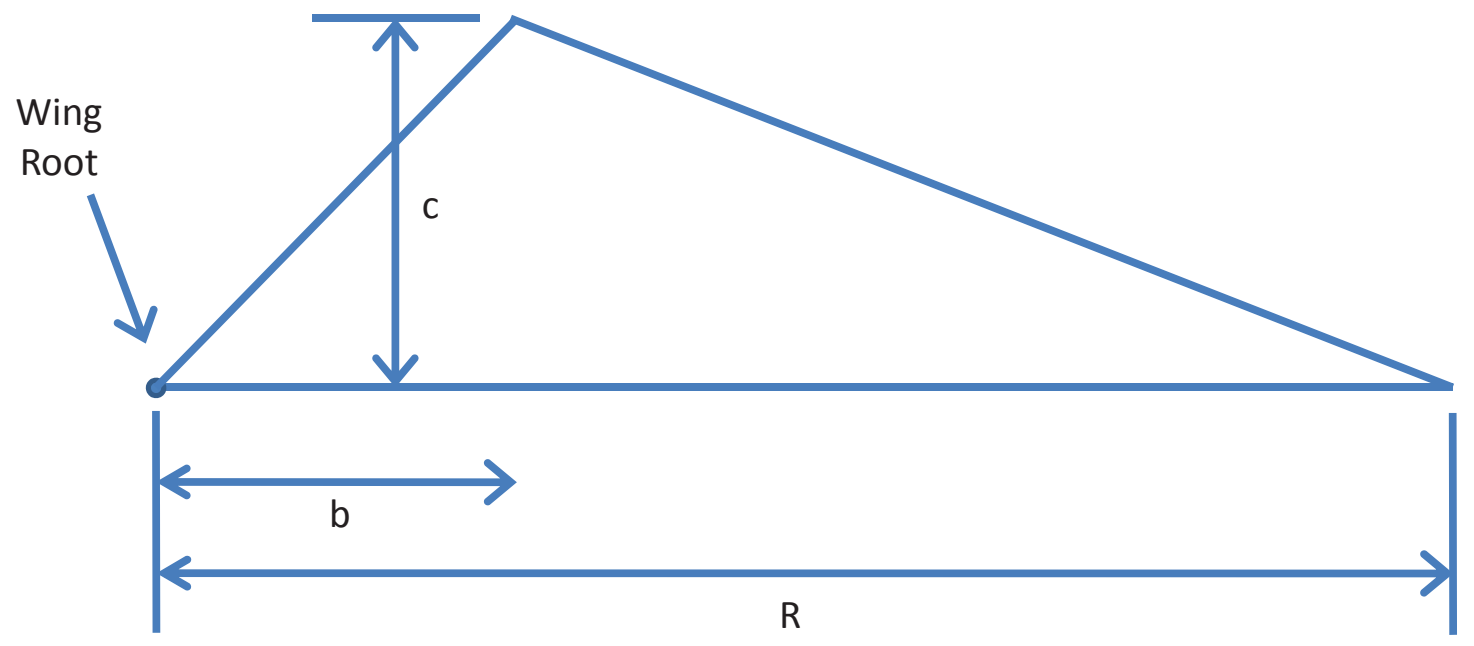

Figure 6: Wing Geometry.

\section{Results}

In this section, time histories of aerodynamic and inertial loads are compared. The inertial loads are calculated according to the expressions shown above. The aerodynamic loads are computed using a blade element model. A full description of the aerodynamic loads can be found in Doman, et. al. ${ }^{8}$

The wing geometry is shown in Figure 6. Wing parameters are displayed in Table 1, while vehicle parameters are shown in Table 2. The trim frequencies are 98.39, 80.33, and $62.22 \frac{\mathrm{rad}}{\mathrm{sec}}$ for cases 1, 2, and 3, respectively. The trim frequency is found be setting the cycle-averaged aerodynamic lift from both wings equal to the weight of the vehicle.

\begin{tabular}{|c|c|c|c|c|c|c|c|}
\hline Case & $b(\mathrm{~mm})$ & $c(\mathrm{~mm})$ & $R(\mathrm{~mm})$ & $I_{A}\left(\mathrm{~m}^{4}\right)$ & Area $\left(\mathrm{m}^{2}\right)$ & AR & WingMass $(\mathrm{mg})$ \\
\hline 1 & 3 & 4 & 15 & $1.395 e^{-9}$ & $3 e^{-5}$ & 7.5 & 0.25 \\
\hline 2 & 3 & 6 & 15 & $2.093 e^{-9}$ & $4.5 e^{-5}$ & 5 & 0.4 \\
\hline 3 & 3 & 15 & 15 & $5.231 e^{-9}$ & $1.125 e^{-4}$ & 2 & 1 \\
\hline
\end{tabular}

Table 1: Wing Parameters.

Figures 7, 8 , and 9 shows the $x, y$, and $z$ body axes inertial and aerodynamic forces and moments for the right and left wings for the geometry of case 1 . As expected, the $x$ body axis inertial force is zero since no out of plane wing motion is considered and the wing flip at the end of each stroke is ignored. There are significant inertial moments about the $x$ body axis, about $50 \%$ greater than those produced by the aerodynamic forces. The inertial loads for the $y$ body axis are also larger than the aerodynamic loads, nearly 4 times the 


\begin{tabular}{|c|c|}
\hline Mass $(\mathrm{mg})$ & 60 \\
\hline$x_{R W R} \& x_{L W R}(\mathrm{~mm})$ & 9 \\
\hline$y_{R W R}(\mathrm{~mm})$ & 2 \\
\hline$z_{R W R} \& z_{L W R}(\mathrm{~mm})$ & 0 \\
\hline$y_{L W R}(\mathrm{~mm})$ & -2 \\
\hline$\alpha(\mathrm{deg})$ & 45 \\
\hline
\end{tabular}

Table 2: Vehicle Parameters.

aerodynamic loads. The moments about the $y$ axis tell a different story. In this case, the aerodynamic moments are larger than the inertial moments. This is due to two factors. Consider a standard cross-product result:

$\mathbf{r} \times \mathbf{F}=\left[\begin{array}{lll}r_{x} & r_{y} & r_{z}\end{array}\right] \times\left[\begin{array}{lll}F_{x} & F_{y} & F_{z}\end{array}\right]=\left(r_{y} F_{z}-r_{z} F_{y}\right) \hat{i}-\left(r_{x} F_{z}-r_{z} F_{x}\right) \hat{j}+\left(r_{x} F_{y}-r_{y} F_{x}\right) \hat{k}$

The $y$ axis moment is the second term in Equation 23, namely, $\left(r_{x} F_{z}-r_{z} F_{x}\right)$. In the calculation of the loads, $r_{x}$ and $r_{z}$ are the same for the aerodynamic and inertial calculations, while $F_{z}$ and $F_{x}$ are different for the aerodynamic and inertial calculations. The aerodynamic force in the $x$ axis direction is nonzero and large, while the inertial force in the $x$ axis direction is identically zero. It should be pointed out that the inertial force in the $x$ axis is zero because the wing flip dynamics have been ignored. The moment arms that affect the pitching moment are $r_{x}$ and $r_{z}$ and $r_{x} \ll r_{z}$. Therefore, the term multiplying $F_{x}$ is large with respect to the term multiplying $F_{z}$. The result is the term $r_{z} F_{x}$ dominates with $F_{x}$ being nonzero for the aerodynamic contribution and zero for the inertial computation. The inertial and aerodynamic forces in the $z$ body axis are similar in magnitude. Again, the aerodynamic moments about the $z$ axis dominate for the same reason as was seen with the $y$ body axis moments.

Now, the case 2 geometry, as shown in Table 1, is utilized. Figures 10, 11, and 12 shows the $x, y$, and $z$ body axes inertial and aerodynamic forces and moments for the right and left wings for the geometry of case 2 . The first interesting aspect is that the body axis force (lift) in the $x$ direction does not change, even when the wing is increased in size. This is because the increase in size is exactly canceled by the decrease in trim frequency, when calculating lift. The inertial moments about the $x$ body axis are slightly larger for case 2 as compared to case 1 . In terms of the $y$ axis, both the inertial forces and moments are larger for case 2 as compared to case 1 . Another interesting aspect is that the aerodynamic moments about the $y$ body axis are different between cases 1 and 2. This is due to the wing geometry changing and the effect it has on the moment arm. For the $z$ body axis, the inertial moments are larger 

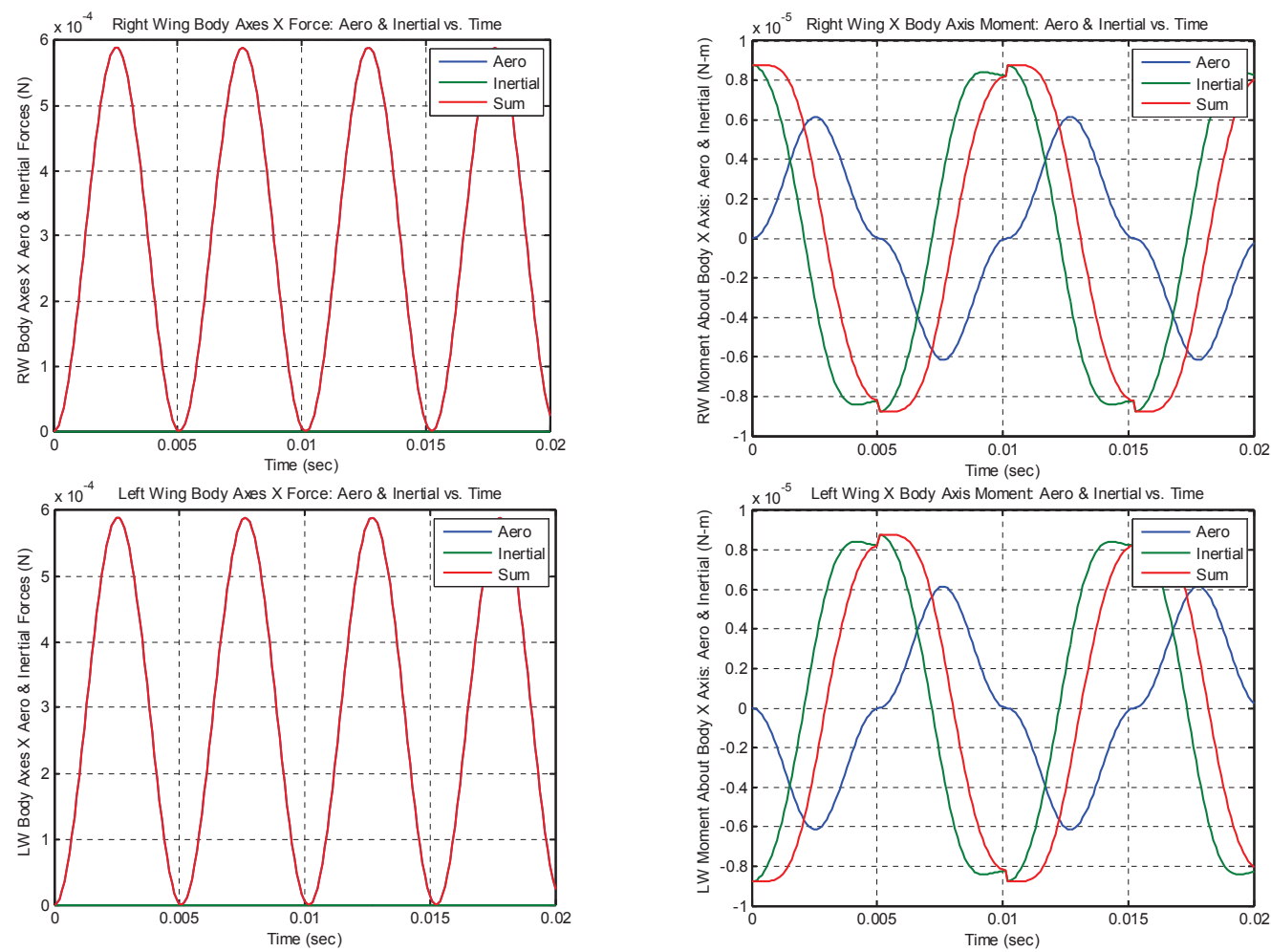

Figure 7: X-Body Axis Inertial and Aerodynamic Forces and Moments Due to Right and Left Wings for Case 1 Geometry.

than those associated with the case 1 geometry, while the inertial forces are only slightly larger.

For case 3, the wing aspect ratio was reduced even further. Similar results are obtained as those discussed when moving from case 1 to case 2 . Both the inertial forces and moments become larger, as can be seen in Figures 13, 14, and 15.

\section{Conclusions}

This work described a method to compute inertial loads associated with a rigid flapping wing. The acceleration of the wing center-of-gravity was computed. Using this acceleration, the inertial forces and moments were calculated. As compared to a blade element aerodynamic model, the inertial forces are larger than the aerodynamic forces, but the inertial moments are smaller than the aerodynamic moments. This is a direct result of the fact that the inertial loads associated with the wing reversal are ignored. The next step in this work would be to incorporate wing flexibility into the model to account for bending and torsion. It is expected that significantly different results will be obtained, as compared to the rigid wing results presented here. Additionally, wing flip dynamics should be incorporated into 

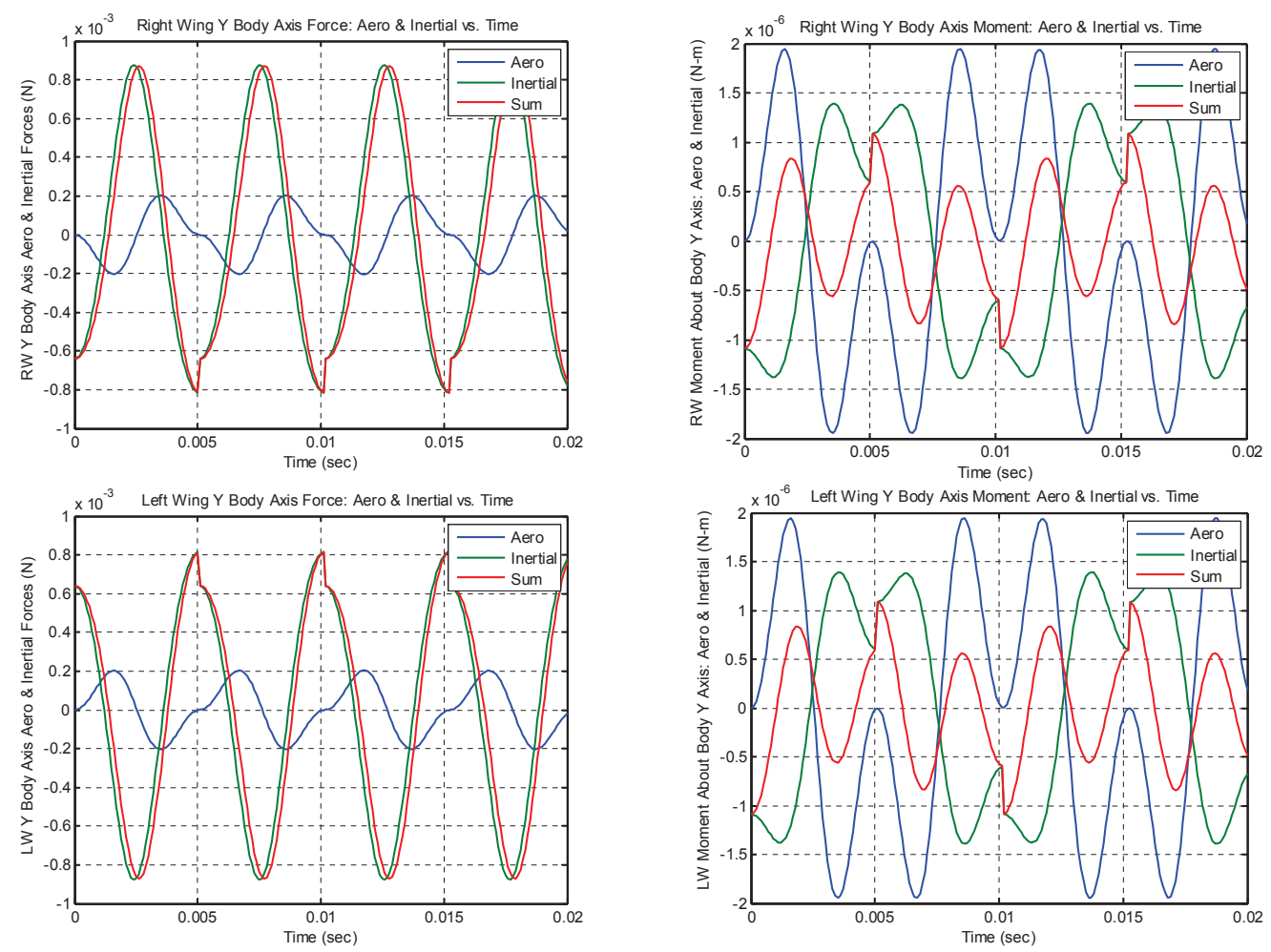

Figure 8: Y-Body Axis Inertial and Aerodynamic Forces and Moments Due to Right and Left Wings for Case 1 Geometry.

the analysis. With the wing flip, inertial forces in the $x$ body axis direction would no longer be zero. Furthermore, experiments using actual wings could be performed to validate the theoretical results.

\section{References}

${ }^{1}$ Sane, S. P. and Dickinson, M. H., "The Control of Flight Force by a Flapping Wing: Lift and Drag Production," The Journal of Experimental Biology, Vol. 204, No. 15, 2001, pp. 2607-2626.

${ }^{2}$ Sane, S. P. and Dickinson, M. H., "The Aerodynamic Effects of Wing Rotation and a Revised QuasiSteady Model of Flapping Flight," The Journal of Experimental Biology, Vol. 205, No. 8, 2002, pp. 1087-1096.

${ }^{3}$ Wood, R. J., "The First Takeoff of a Biologically Inspired At-Scale Robotic Insect," IEEE Transactions on Robotics, Vol. 24, No. 2, 2008, pp. 341-347.

${ }^{4}$ Wood, R. J., "Design, Fabrication, and Analysis of a 3DOF, 3cm Flapping-Wing MAV," Proceedings of the 2007 IEEE/RSJ International Conference on Intelligent Robots and Systems, Oct. 2007.

${ }^{5}$ Ellington, C. P., "The Aerodynamics of Hovering Insect Flight. I. The Quasi-Steady Analysis," Philosophical Transactions of the Royal Society of London: Series B: Biological Series, Vol. 305, 1984.

${ }^{6}$ Dudley, R. and Ellington, C. P., "Mechanics of Forward Flight in Bumblebees I. Kinematics and Morphology," Journal of Experimental Biology, Vol. 148, No. 1, 1990, pp. 19-52. 

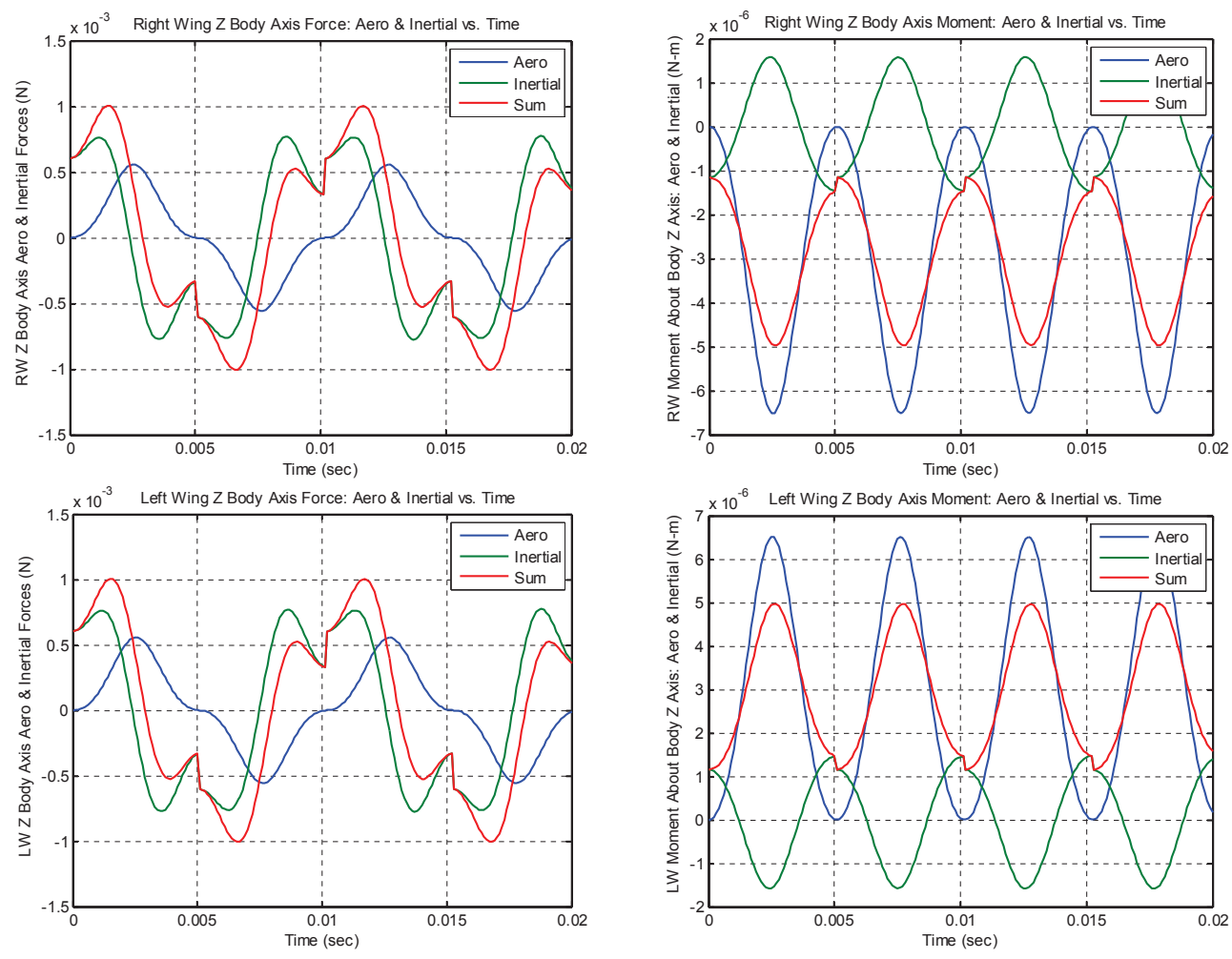

Figure 9: Z-Body Axis Inertial and Aerodynamic Forces and Moments Due to Right and Left Wings for Case 1 Geometry.

${ }^{7}$ Dudley, R. and Ellington, C. P., "Mechanics of Forward Flight in Bumblebees II. Quasi-Steady Lift and Power Requirements," Journal of Experimental Biology, Vol. 148, No. 1, 1990, pp. 53-88.

${ }^{8}$ Doman, D. B., Oppenheimer, M. W., and Sigthorsson, D. O., "Wingbeat Shape Modulation for Flapping-Wing Micro-Air Vehicle Control During Hover," Journal of Guidance, Control and Dynamics, Vol. 33, No. 3, 2010, pp. 724-739.

${ }^{9}$ Deng, X., Schenato, L., and Sastry, S. S., "Flapping Flight for Biomimetic Robotic Insects: Part II Flight Control Design," IEEE Trans. on Robotics, Vol. 22, No. 4, 2006, pp. 789-803.

${ }^{10}$ Shyy, W., Lain, Y., Tang, J., Viieru, D., and Lui, H., Aerodynamics of Low Reynolds Number Flyers, Cambridge University Press, New York, NY, 2008, pp. 6.

${ }^{11}$ Ellington, C. P., "The Aerodynamics of Hovering Insect Flight. II. Morphological Parameters," Philosophical Transactions of the Royal Society of London: Series B: Biological Series, Vol. 305, 1984.

${ }^{12}$ Ellington, C. P., "The Aerodynamics of Hovering Insect Flight. III. Kinematics," Philosophical Transactions of the Royal Society of London: Series B: Biological Series, Vol. 305, 1984.

${ }^{13}$ Ellington, C. P., "The Aerodynamics of Hovering Insect Flight. IV. Aerodynamic Mechanisms," Philosophical Transactions of the Royal Society of London: Series B: Biological Series, Vol. 305, 1984.

${ }^{14}$ Ellington, C. P., "The Aerodynamics of Hovering Insect Flight. V. A Vortex Theory," Philosophical Transactions of the Royal Society of London: Series B: Biological Series, Vol. 305, 1984.

${ }^{15}$ Ellington, C. P., "The Aerodynamics of Hovering Insect Flight. VI. Lift and Power Requirements," Philosophical Transactions of the Royal Society of London: Series B: Biological Series, Vol. 305, 1984. 

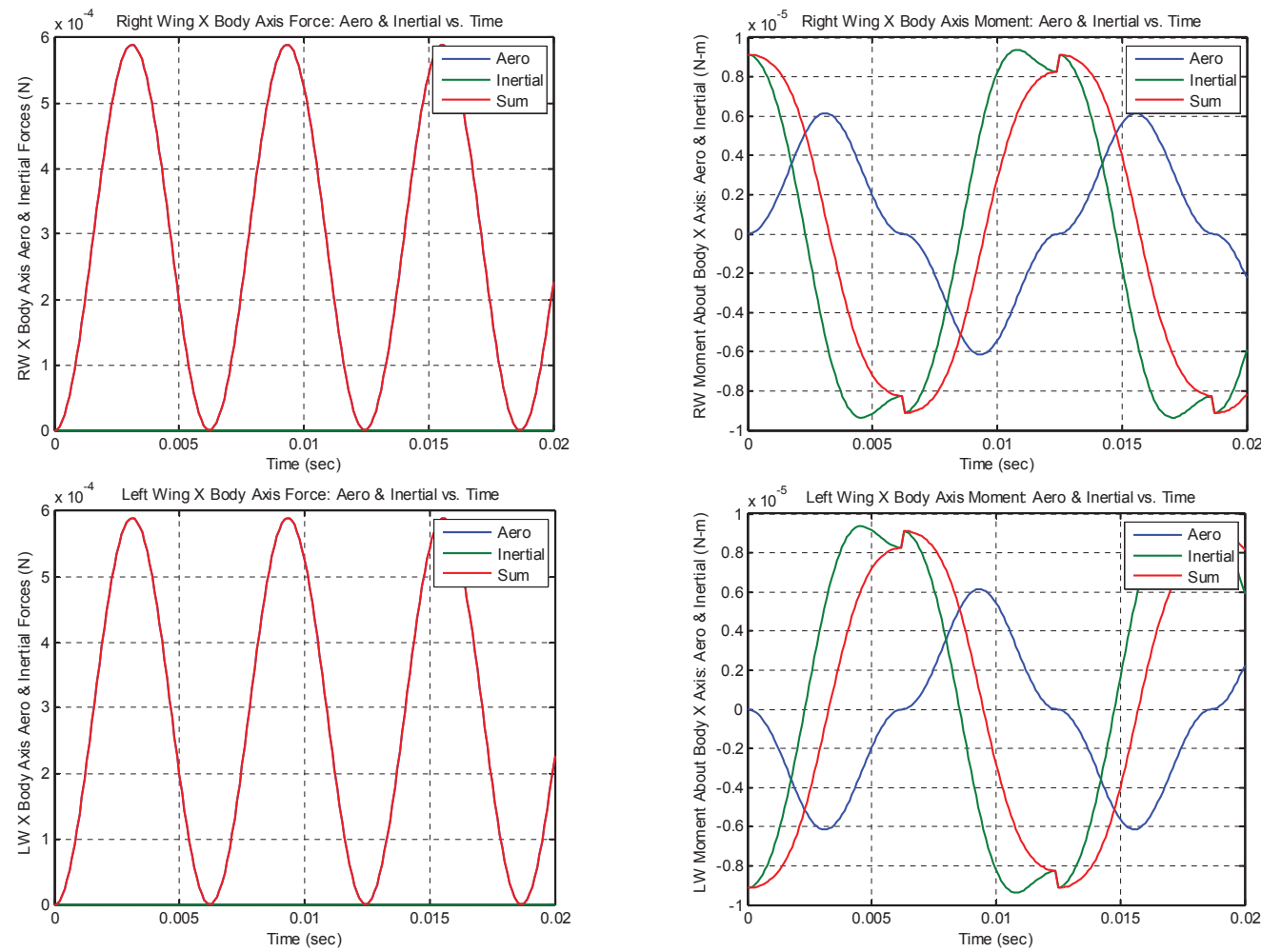

Figure 10: X-Body Axis Inertial and Aerodynamic Forces and Moments Due to Right and Left Wings for Case 2 Geometry.

${ }^{16}$ Deng, X., Schenato, L., Wu, W. C., and Sastry, S. S., "Flapping Flight for Biomimetic Robotic Insects: Part I - System Modeling," IEEE Trans. on Robotics, Vol. 22, No. 4, 2006, pp. 776-788.

${ }^{17}$ Żbikowski, R., "On Aerodynamic Modelling of an Insect-Like Flapping Wing in Hover for Micro Air Vehicles," Philosophical Transactions of the Royal Society of London: Series A, Vol. 360, 2002.

${ }^{18}$ Sane, S. P., "The Aerodynamics of Insect Flight," Journal of Experimental Biology, Vol. 206, 2003, pp. 4191-4208.

${ }^{19}$ Stevens, B. L. and Lewis, F. L., Aircraft Control and Simulation, John Wiley \& Sons, Inc., 2003. 

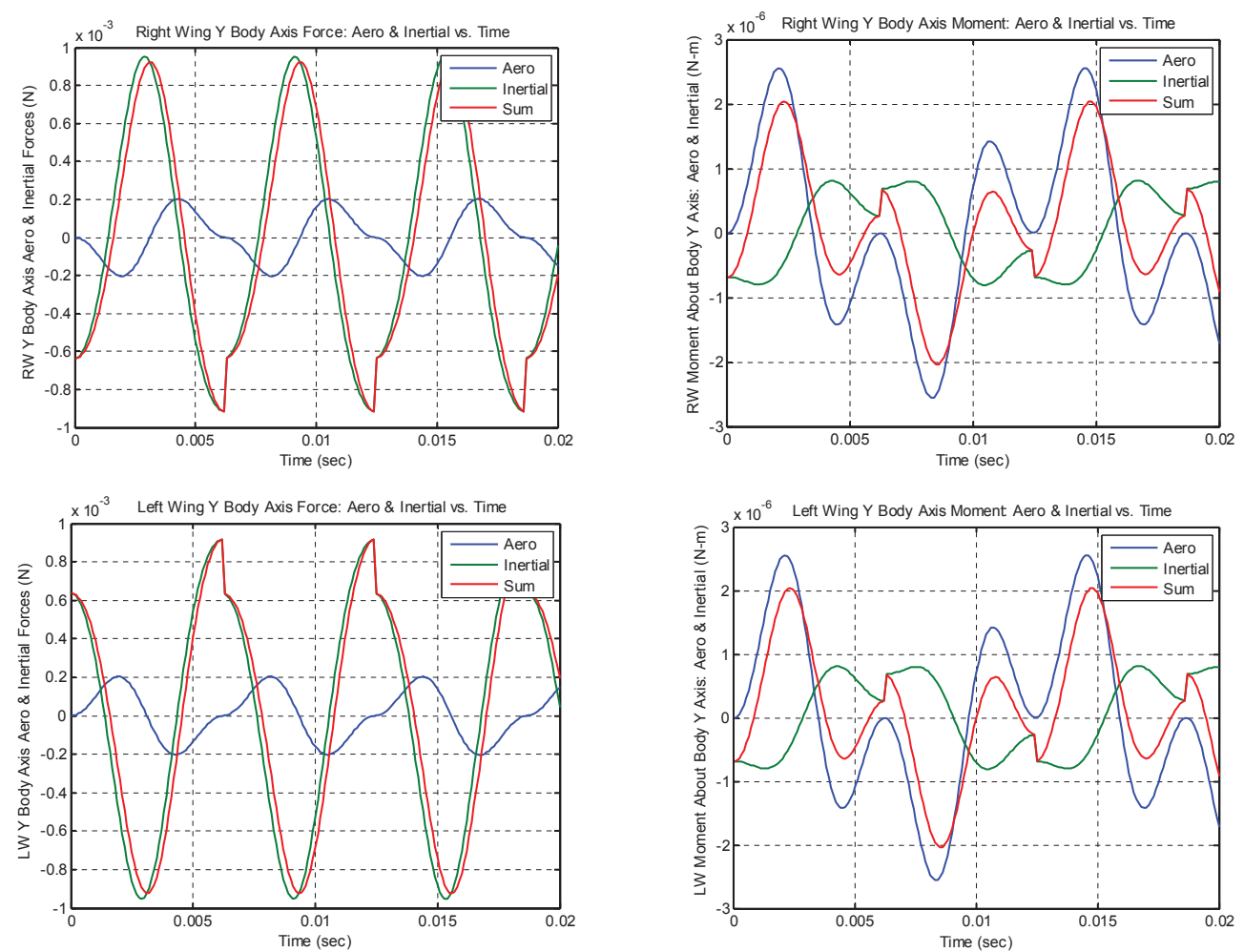

Figure 11: Y-Body Axis Inertial and Aerodynamic Forces and Moments Due to Right and Left Wings for Case 2 Geometry. 

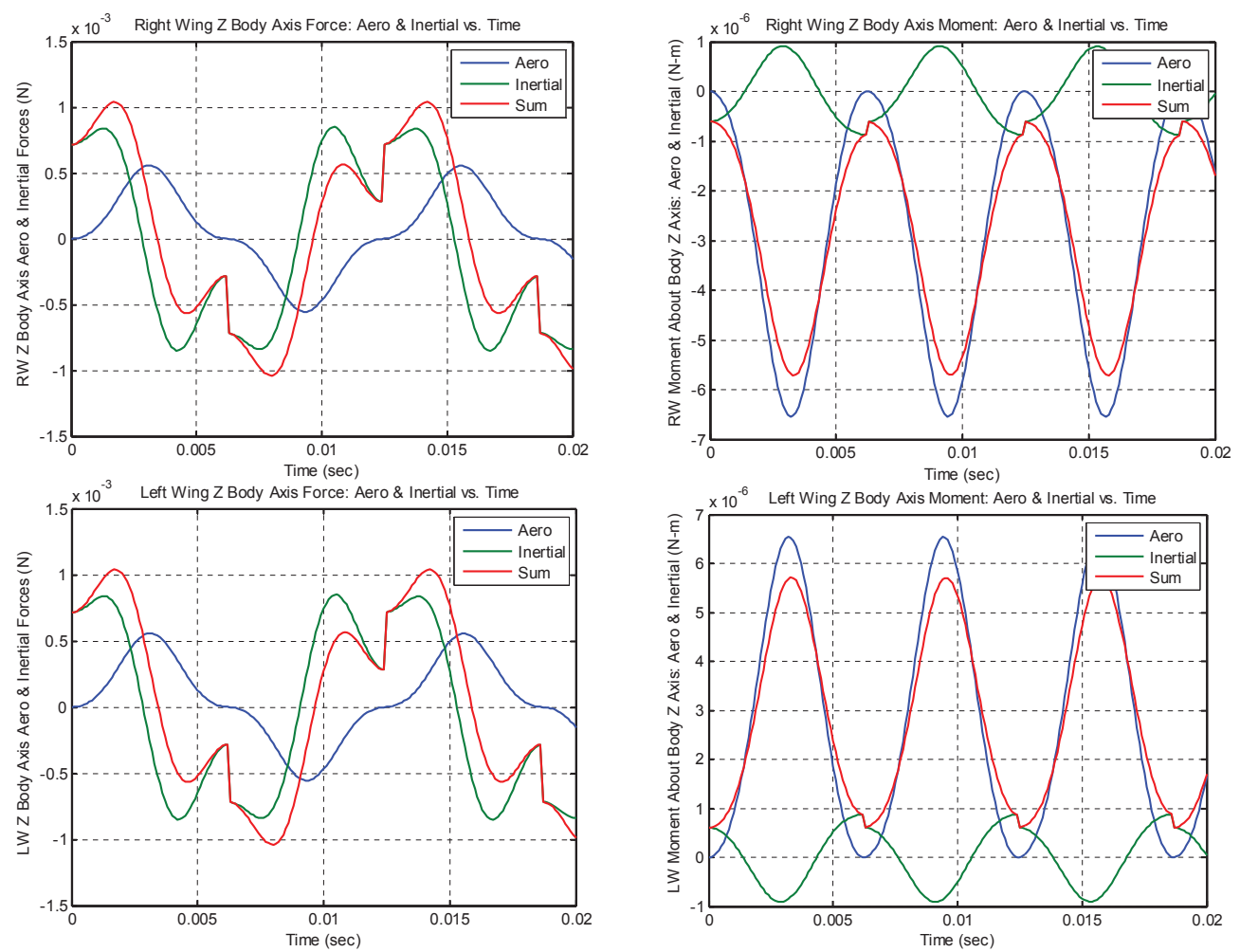

Figure 12: Z-Body Axis Inertial and Aerodynamic Forces and Moments Due to Right and Left Wings for Case 2 Geometry. 

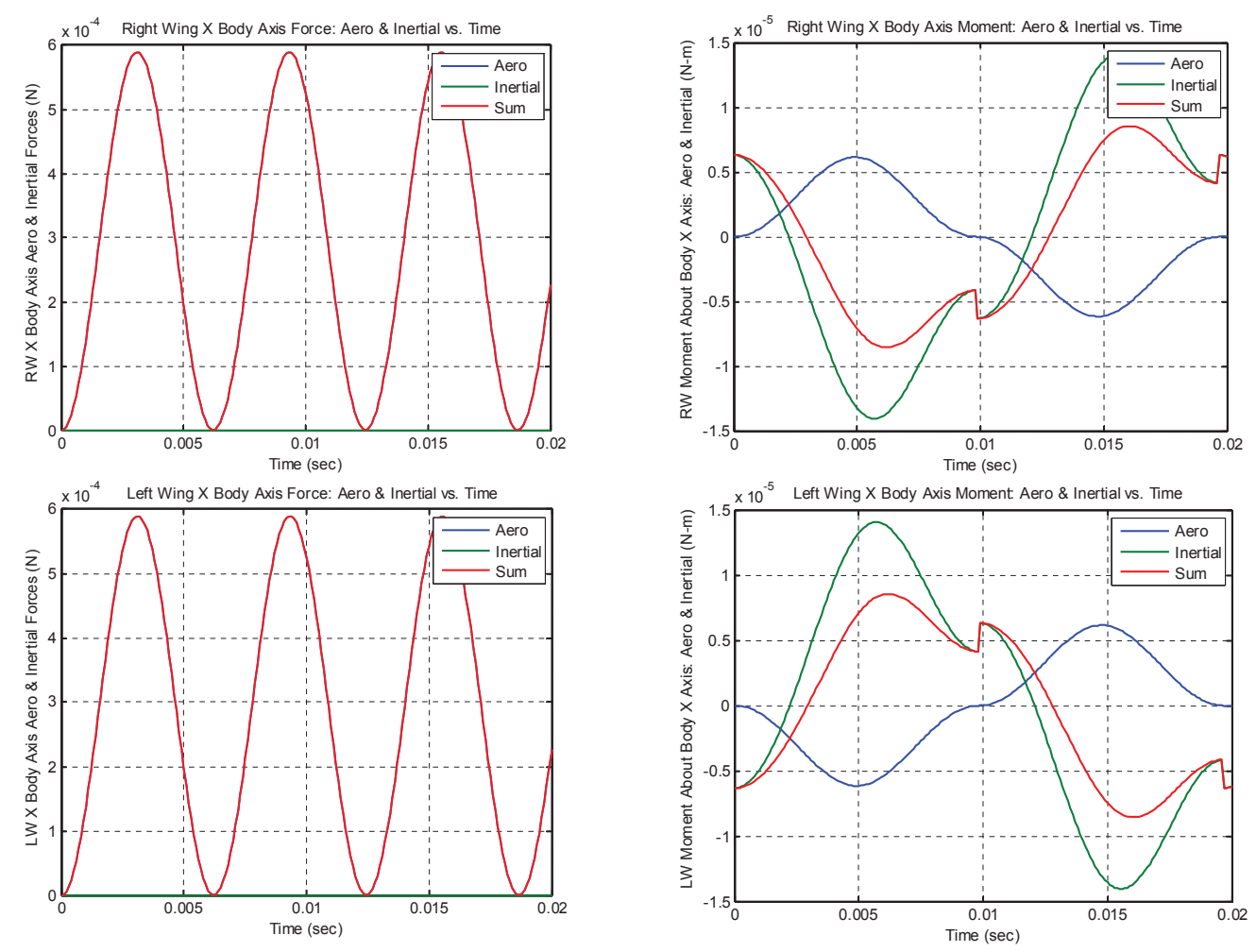

Figure 13: X-Body Axis Inertial and Aerodynamic Forces and Moments Due to Right and Left Wings for Case 3 Geometry. 

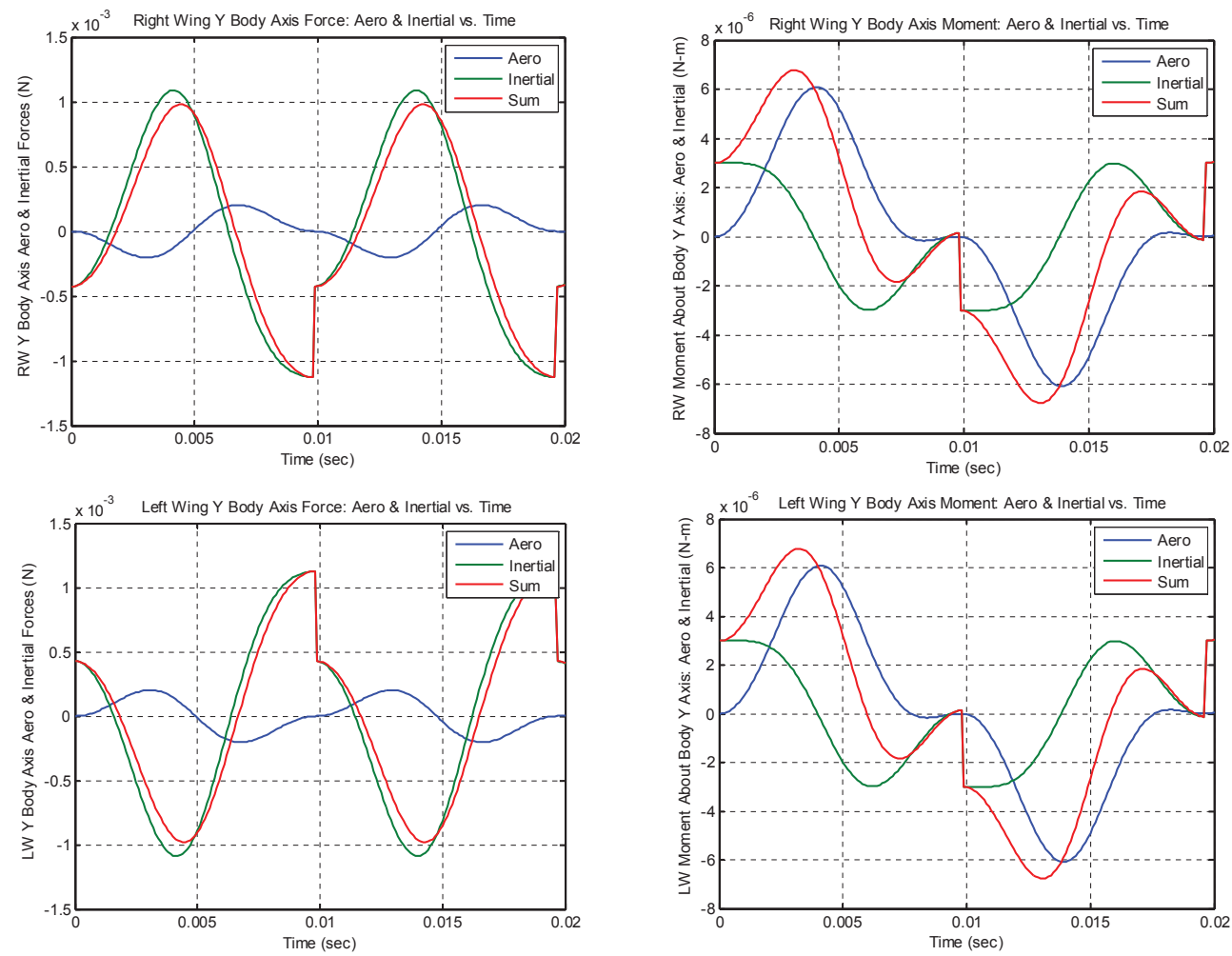

Figure 14: Y-Body Axis Inertial and Aerodynamic Forces and Moments Due to Right and Left Wings for Case 3 Geometry. 

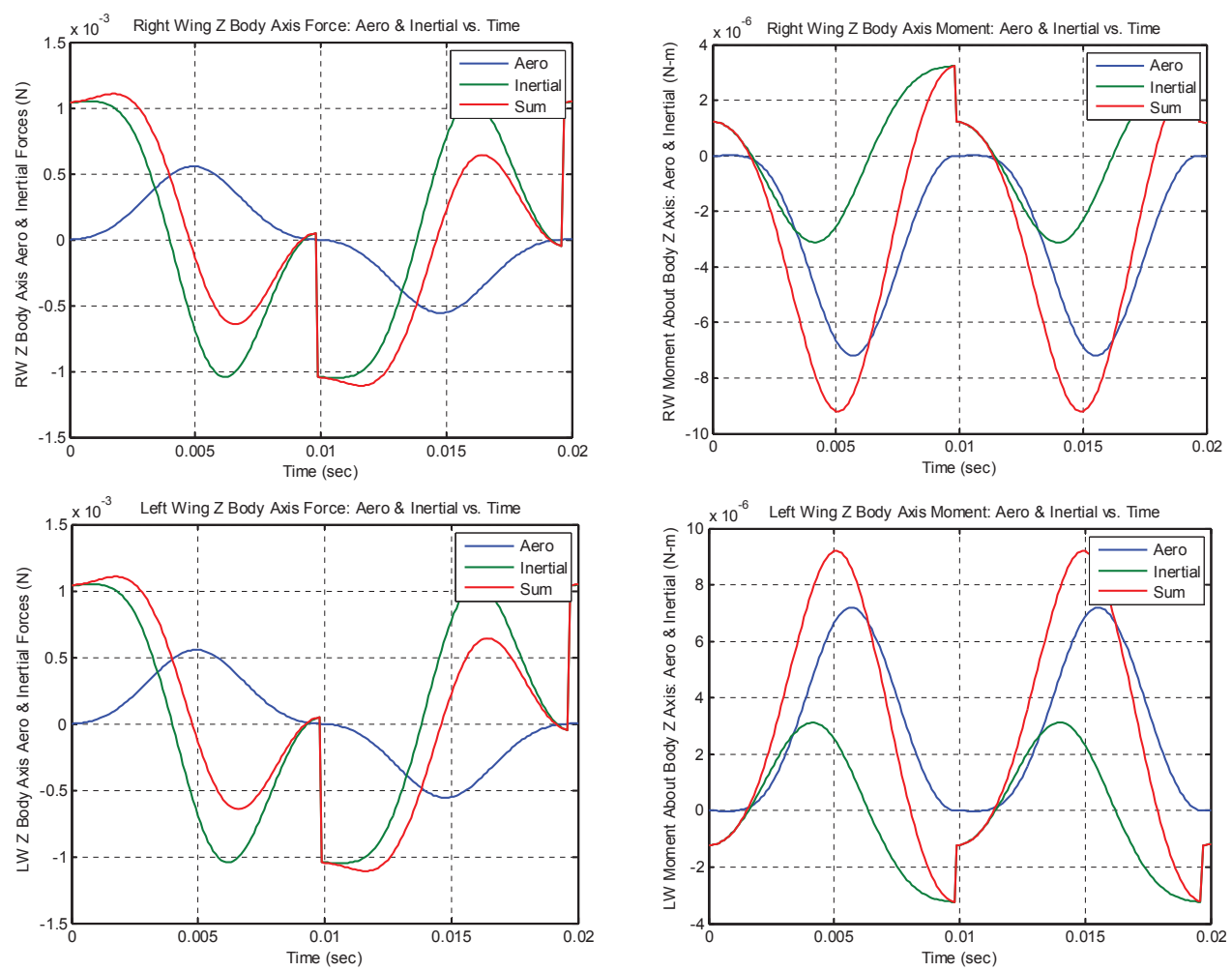

Figure 15: Z-Body Axis Inertial and Aerodynamic Forces and Moments Due to Right and Left Wings for Case 3 Geometry. 